\title{
(The) Nature(s) of Science(s) and (the) Scientific $\operatorname{Method}(\mathbf{s})$
}

\author{
Kostas Kampourakis ${ }^{1}$
}

Published online: 20 January 2016

(c) Springer Science+Business Media Dordrecht 2016

In recent years, there has been a lively discussion and debate on what students should learn about "nature of science" (hereafter NOS). The participants in this debate have disagreed about almost everything: whether there is a single "NOS" or more than one, whether we should talk of science in general or refer to several sciences in particular, whether there are some common methods that all scientists use or whether these methods differ significantly among the various disciplines. On the one hand, several educators have advocated teaching about some general aspects of NOS and have provided empirical research that has shown that students can understand them. On the other hand, some science educators and philosophers have argued that there are no general aspects of science and that students should not be taught about some aspects but not others, because this produces a distorted image of science. I have discussed the arguments of the opposing sides in these debates elsewhere (see an upcoming issue of the Journal of Research in Science Teaching), and I have suggested a possible way forward. In this editorial, I want to note that it might be useful to approach teaching and learning NOS from the perspective of research on conceptual change.

I think that there has been some confusion regarding the main aim of NOS teaching. In some cases, it seems that the aim is to describe what science is, how it is done and in what ways and why it differs from other human activities. This approach implies that it is possible to find criteria adequate for demarcating science from pseudoscience, or nonscience more generally. However, demarcation is not only a difficult and philosophically challenging task, but it is also questionable as an aim for NOS teaching. Indeed, why would we want to make science distinct from other human activities? Science is about the study of the natural world, and about understanding and representing its phenomena. Science is about making inferences, drawing conclusions and developing theories, models and explanations on the basis of empirical data. But scientific investigations are not the only investigations that are based on empirical data-just think of the work of the fictional

Kostas Kampourakis

Kostas.Kampourakis@unige.ch

1 University of Geneva, Pavillon Mail, 40 Boulevard du Pont-d'Arve, 1211 Geneva, Switzerland 
detective Sherlock Holmes. Scientific investigation and criminal investigation share some features, but of course differ in others (e.g., the object of study). Is it so important to compare the two and find criteria for demarcating between them? In my view, this is not the main problem.

What I view as the main challenge, and one that should be a central goal of NOS teaching, is how to effectively address students' conceptions of science, which stem from images imposed by culture, media, and other social representations of science. For example, TV series presenting how forensic investigators work often provide a distorted image of science. The analysis of DNA extracted from a blood drop is presented as sufficient for establishing whether a suspect is innocent or guilty of a crime. DNA analysis is also presented as swift, reliable, and unproblematic despite numerous practical problems. Similarly, whereas in medicine conclusions are often "grey," many patients expect physicians to provide them with a "black" or a "white" conclusion about a conditionbecause they think that this is possible, although often it is not. And companies selling direct-to-consumer genetic tests sometimes make bold claims, ranging from whether or not one will have a particular disease to specifying one's ideal partner. Science is often portrayed as authoritative and certain, and confidence in science can be lost when it fails to make authoritative and certain claims.

Against these everyday conceptions, we need to portray science as a human and social process; we need to give our students an authentic view of how science has been and is currently being done. This can be achieved in various ways, but research suggests that teaching should be explicit and reflective. Prior to such teaching, students' conceptions should be uncovered and analyzed in detail. Teaching about NOS should start from these conceptions and not from what we think that students ought to know about science. Some of these conceptions have already been documented in detail, but perhaps we need to probe students' thinking in greater detail. Teaching and learning for conceptual change has been the focus of research for various topics in science education, but not that much in relation to NOS. I suggest that we need to move away from debates about what we should teach about NOS, and toward investigations of teaching and learning about NOS through the lens of conceptual change research. This approach could trace learning pathways that focus on students' NOS conceptions and understandings from a developmental perspective.

Ten years ago, in an article published in this journal titled "Learners' Responses to the Demands of Conceptual Change: Considerations for Effective Nature of Science Instruction," Michael Clough suggested that "A conceptual change framework helps make sense of the difficulties students often have developing robust understandings of the NOS that can be applied in a variety of settings. In doing so, it explains the importance of explicit and reflective NOS instruction, but also raises additional issues to consider in effective NOS instruction." We currently know a lot about students' and teachers' (mis)understandings of and difficulties in understanding NOS. I think that it is now time to envision the challenges of teaching NOS through the lens of conceptual change. Science \& Education would welcome research on the benefits and drawbacks of approaching NOS from a conceptual change perspective. 